Bull. Korean Math. Soc. 47 (2010), No. 6, pp. 1235-1250

DOI 10.4134/BKMS.2010.47.6.1235

\title{
THREE SOLUTIONS TO A CLASS OF NEUMANN DOUBLY EIGENVALUE ELLIPTIC SYSTEMS DRIVEN BY A $\left(p_{1}, \ldots, p_{n}\right)$-LAPLACIAN
}

\author{
Ghasem A. Afrouzi, Shapour Heidarkhani, and Donal O'Regan
}

\begin{abstract}
In this paper we establish the existence of at least three weak solutions for Neumann doubly eigenvalue elliptic systems driven by a $\left(p_{1}, \ldots, p_{n}\right)$-Laplacian. Our main tool is a recent three critical points theorem of B. Ricceri.
\end{abstract}

\section{Introduction}

Here and in the sequel, $\Omega \subset \mathbb{R}^{N}(N \geq 1)$ is a non-empty bounded open set with a boundary $\partial \Omega$ of class $C^{1}, p_{i} \geq 2$ and $a_{i} \in L^{\infty}(\Omega)$ with $\operatorname{essinf}_{\Omega} a_{i}>0$ for $1 \leq i \leq n$.

In this paper we are interested in multiplicity results for the following Neumann elliptic system

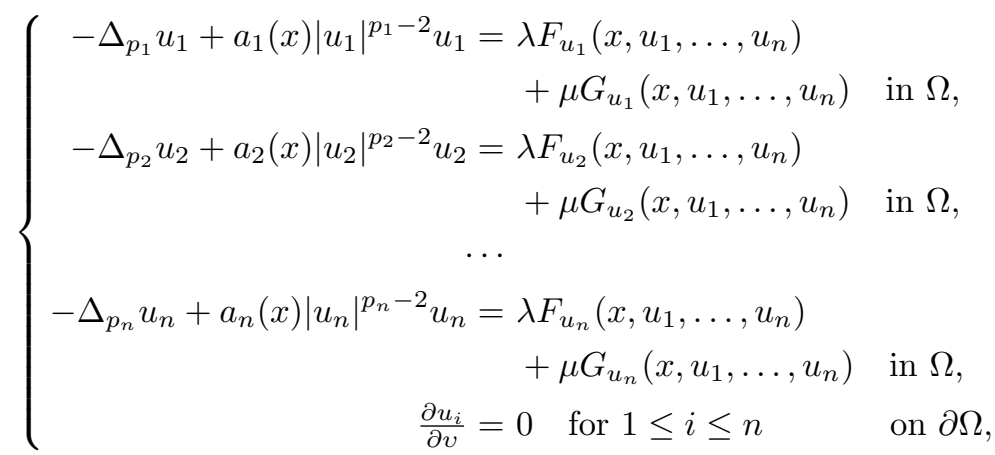

where $\Delta_{p_{i}} u_{i}=\operatorname{div}\left(\left|\nabla u_{i}\right|^{p_{i}-2} \nabla u_{i}\right)$ is the $p_{i}$-Laplacian operator, $p_{i}>N$ for $1 \leq i \leq n, \lambda, \mu>0, F: \Omega \times \mathbb{R}^{n} \rightarrow \mathbb{R}$ is a function such that $F\left(\cdot, t_{1}, \ldots, t_{n}\right)$ is continuous in $\Omega$ for all $\left(t_{1}, \ldots, t_{n}\right) \in \mathbb{R}^{n}$ and $F(x, \cdot, \ldots, \cdot)$ is $C^{1}$ in $\mathbb{R}^{n}$ for almost every $x \in \Omega, G: \Omega \times \mathbb{R}^{n} \rightarrow \mathbb{R}$ is a function such that $G\left(\cdot, t_{1}, \ldots, t_{n}\right)$ is measurable in $\Omega$ for all $\left(t_{1}, \ldots, t_{n}\right) \in \mathbb{R}^{n}$ and $G(x, \cdot, \ldots, \cdot)$ is $C^{1}$ in $\mathbb{R}^{n}$ for

Received April 14, 2009.

2000 Mathematics Subject Classification. 35J65, 34A15.

Key words and phrases. three solutions, critical point, $\left(p_{1}, \ldots, p_{n}\right)$-Laplacian, multiplicity results, Neumann problem. 
almost every $x \in \Omega, F_{u_{i}}$ and $G_{u_{i}}$ denotes the partial derivative of $F$ and $G$ with respect to $u_{i}$, respectively, and $v$ is the outward unit normal to $\partial \Omega$.

A weak solution of $(1)$ is any $u=\left(u_{1}, u_{2}, \ldots, u_{n}\right) \in W^{1, p_{1}}(\Omega) \times W^{1, p_{2}}(\Omega) \times$ $\cdots \times W^{1, p_{n}}(\Omega)$ such that

$$
\begin{aligned}
& \int_{\Omega} \sum_{i=1}^{n}\left|\nabla u_{i}(x)\right|^{p_{i}-2} \nabla u_{i}(x) \nabla v_{i}(x) d x+\int_{\Omega} \sum_{i=1}^{n} a_{i}(x)\left|u_{i}(x)\right|^{p_{i}-2} u_{i}(x) v_{i}(x) d x \\
& -\left(\lambda \int_{\Omega} \sum_{i=1}^{n} F_{u_{i}}\left(x, u_{1}(x), \ldots, u_{n}(x)\right) v_{i}(x) d x+\mu \int_{\Omega} \sum_{i=1}^{n} G_{u_{i}}\left(x, u_{1}(x), \ldots, u_{n}(x)\right) v_{i}(x) d x\right)=0
\end{aligned}
$$

for every $\left(v_{1}, v_{2}, \ldots, v_{n}\right) \in W^{1, p_{1}}(\Omega) \times W^{1, p_{2}}(\Omega) \times \cdots \times W^{1, p_{n}}(\Omega)$.

In the literature many papers $[5,10,12-14,16,17,22,23]$ discuss quasilinear elliptic systems. For example in [12] the authors studied a class of quasilinear elliptic systems involving the $p$-Laplacian operator where the right hand side is closely related to the critical Sobolev exponent and they proved the existence of at least one nontrivial solution under suitable assumptions on the nonlinearities. In [10], Y. Bozhkova and E. Mitidieri using the fibering method, introduced by Pohozaer, established the existence of multiple solutions for a Dirichlet problem associated with a quasilinear system involving $(p, q)$-Laplacian operators. In [16], A. Kristály using an abstract critical point result of B. Ricceri established the existence of an interval $\Lambda \subseteq[0,+\infty[$ such that for each $\lambda \in \Lambda$ the quasilinear elliptic system

$$
\left\{\begin{aligned}
-\Delta_{p} u & =\lambda F_{u}(x, u, v) & & \text { in } \Omega, \\
-\Delta_{q} v & =\lambda F_{v}(x, u, v) & & \text { in } \Omega, \\
u=v & =0 & & \text { on } \partial \Omega,
\end{aligned}\right.
$$

where $\Omega$ is a strip-like domain and $\lambda>0$ is a parameter, has at least two distinct nontrivial solutions. In [23], the authors studied the Nehari manifold for a class of quasilinear elliptic systems involving a pair of $(p, q)$-Laplacian operators and a parameter, and proved the existence of a nonnegative solution for the system by discussing properties of the Nehari manifold, and they obtained global bifurcation results. We also refer the reader to $[1,2,4,6-9,11,17-19]$ where the three critical points theorem of B. Ricceri [20] is used. Chun Li and Chun-Lei Tang in [17] established the existence of an interval $\Lambda \subseteq[0,+\infty[$ and a positive real number $\rho$ such that for each $\lambda \in \Lambda$ problem (2) admits at least three weak solutions whose norms in $W_{0}^{1, p}(\Omega) \times W_{0}^{1, q}(\Omega)$ are less than $\rho$. In [1] a similar result was obtained for the quasilinear elliptic system

$$
\left\{\begin{array}{cc}
\Delta_{p_{1}} u_{1}+\lambda F_{u_{1}}\left(x, u_{1}, u_{2}, \ldots, u_{n}\right)=0 & \text { in } \Omega, \\
\Delta_{p_{2}} u_{2}+\lambda F_{u_{2}}\left(x, u_{1}, u_{2}, \ldots, u_{n}\right)=0 & \text { in } \Omega, \\
\cdots & \\
\Delta_{p_{n}} u_{n}+\lambda F_{u_{n}}\left(x, u_{1}, u_{2}, \ldots, u_{n}\right)=0 & \text { in } \Omega, \\
u_{i}=0 \text { for } 1 \leq i \leq n & \text { on } \partial \Omega .
\end{array}\right.
$$


In [8], G. Bonanno and P. Candito using Ricceri's three critical points theorem, proved the existence of an interval $\Lambda \subseteq[0,+\infty[$ and a positive real number $q$ such that for each $\lambda \in \Lambda$ the problem

$$
\left\{\begin{aligned}
-\Delta_{p} u+a_{1}(x)|u|^{p-2} u_{1} & =\lambda f(x, u) & & \text { in } \Omega, \\
\frac{\partial u}{\partial v} & =0 & & \text { on } \partial \Omega,
\end{aligned}\right.
$$

where $\Omega \subset \mathbb{R}^{N}(N \geq 1)$ is a nonempty bounded open set with a boundary $\partial \Omega$ of class $C^{1}, a \in L^{\infty}(\Omega)$ with $\operatorname{essinf}_{\Omega} a>0, p>N, \lambda>0$ and $f: \Omega \times \mathbb{R} \rightarrow \mathbb{R}$ is a continuous function, admits at least three weak solutions whose norms in $W^{1, p}(\Omega)$ are less than $q$. Finally, B. Ricceri in [21] extended and revisited the three critical point theorem he obtained in [20], and in [15] the results were used to study the problem

$$
\left\{\begin{array}{l}
u^{\prime \prime}+(\lambda f(t, u)+g(u)) h\left(t, u^{\prime}\right)=\mu p(t, u) h\left(t, u^{\prime}\right) \quad \text { in }(a, b), \\
u(a)=u(b)=0,
\end{array}\right.
$$

where $f:[a, b] \times \mathbb{R} \rightarrow \mathbb{R}$ is a continuous function, $g: R \rightarrow R$ is a Lipschitz continuous function, $\lambda>0, h:[a, b] \times \mathbb{R} \rightarrow \mathbb{R}$ is a bounded function, $\mu>$ 0 and $p:[a, b] \times \mathbb{R} \rightarrow \mathbb{R}$ is a $L^{1}$-Carathéodory function for every $(t, u) \in[a, b] \times \mathbb{R}$.

\section{Preliminaries}

First we recall the three critical points theorem [21].

Theorem 1. Let $X$ be a reflexive real Banach space, $I \subseteq \mathbb{R}$ an interval, $\Phi$ : $X \rightarrow \mathbb{R}$ a sequentially weakly lower semicontinuous $C^{1}$ functional bounded on each bounded subset of $X$ whose derivative admits a continuous inverse on $X^{*}$ and $J: X \rightarrow \mathbb{R}$ a $C^{1}$ functional with compact derivative. Assume that

$$
\lim _{\|x\| \rightarrow+\infty}(\Phi(x)+\lambda J(x))=+\infty
$$

for all $\lambda \in I$, and that there exists $\rho \in \mathbb{R}$ such that

$$
\sup _{\lambda \in I} \inf _{x \in X}(\Phi(x)+\lambda(J(x)+\rho))<\inf _{x \in X} \sup _{\lambda \in I}(\Phi(x)+\lambda(J(x)+\rho)) .
$$

Then, there exist a non-empty open set interval $A \subseteq I$ and a positive real number $q$ with the following property: for every $\lambda \in A$ and every $C^{1}$ functional $\Psi: X \rightarrow \mathbb{R}$ with compact derivative, there exists $\delta>0$ such that, for each $\mu \in[0, \delta]$, the equation

$$
\Phi^{\prime}(u)+\lambda J^{\prime}(u)+\mu \Psi^{\prime}(u)=0
$$

has at least three solutions in $X$ whose norms are less than $q$.

In the proof of our main result we use the next result to verify the minimax inequality in Theorem 1 . 
Proposition 1 ([Bonanno, 7]). Let $X$ be a non-empty set and $\Phi, J$ two real functions on $X$. Assume that $\Phi(x) \geq 0$ for every $x \in X$ and there exists $u_{0} \in X$ such that $\Phi\left(u_{0}\right)=J\left(u_{0}\right)=0$. Further, assume that exist $u_{1} \in X, r>0$ such that

$\left(\kappa_{1}\right) \Phi\left(u_{1}\right)>r$,

$\left(\kappa_{2}\right) \sup _{\Phi(x)<r}(-J(x))<r \frac{-J\left(u_{1}\right)}{\Phi\left(u_{1}\right)}$.

Then, for every $\nu>1$ and for every $\rho \in R$ satisfying

$$
\sup _{\Phi(x)<r}(-J(x))+\frac{r \frac{-J\left(u_{1}\right)}{\Phi\left(u_{1}\right)}-\sup _{\Phi(x)<r}(-J(x))}{\nu}<\rho<r \frac{-J\left(u_{1}\right)}{\Phi\left(u_{1}\right)},
$$

one has

$$
\sup _{\lambda \in \mathbb{R}} \inf _{x \in X}(\Phi(x)+\lambda(J(x)+\rho))<\inf _{x \in X} \sup _{\lambda \in[0, \sigma]}(\Phi(x)+\lambda(J(x)+\rho)),
$$

where

$$
\sigma=\frac{\nu r}{r \frac{-J\left(u_{1}\right)}{\Phi\left(u_{1}\right)}-\sup _{\Phi(x)<r}(-J(x))} .
$$

In order to apply Theorem 1 to our problem, let $X$ be the Cartesian product of the $n$ Sobolev spaces $W^{1, p_{1}}(\Omega), W^{1, p_{2}}(\Omega), \ldots, W^{1, p_{n}}(\Omega)$, i.e., $X=$ $W^{1, p_{1}}(\Omega) \times W^{1, p_{2}}(\Omega) \times \cdots \times W^{1, p_{n}}(\Omega)$ with the norm

$$
\left\|\left(u_{1}, u_{2}, \ldots, u_{n}\right)\right\|=\left\|u_{1}\right\|+\left\|u_{2}\right\|+\cdots+\left\|u_{n}\right\|,
$$

where $\left\|u_{i}\right\|=\left(\int_{\Omega}\left(\left|\nabla u_{i}(x)\right|^{p_{i}}+a_{i}(x)\left|u_{i}(x)\right|^{p_{i}}\right) d x\right)^{1 / p_{i}}$ for $1 \leq i \leq n$.

Put

$$
c=\max \left\{\sup _{u_{i} \in W^{1, p_{i}}(\Omega) \backslash\{0\}} \frac{\max _{x \in \bar{\Omega}}\left|u_{i}(x)\right|^{p_{i}}}{\| u_{i}||^{p_{i}}} ; 1 \leq i \leq n\right\} .
$$

Since $p_{i}>N$ for $1 \leq i \leq n$, one has $c<+\infty$. It follows from Proposition 4.1 of [3] that

$$
\sup _{u_{i} \in W^{1, p_{i}(\Omega) \backslash\{0\}}} \frac{\max _{x \in \bar{\Omega}}\left|u_{i}(x)\right|^{p_{i}}}{\left\|u_{i}\right\|^{p_{i}}}>\frac{1}{\left\|a_{i}\right\|_{1}}, \quad 1 \leq i \leq n,
$$

where $\left\|a_{i}\right\|_{1}=\int_{\Omega}\left|a_{i}(x)\right| d x$ for $1 \leq i \leq n$, and so $\frac{1}{\left\|a_{i}\right\|_{1}} \leq c$ for $1 \leq i \leq n$. In addition, if $\Omega$ is convex, it is known [3] that

$$
\begin{gathered}
\sup _{u_{i} \in W^{1, p_{i}}(\Omega) \backslash\{0\}} \frac{\max _{x \in \bar{\Omega}}\left|u_{i}(x)\right|}{\left\|u_{i}\right\|} \\
\leq 2^{\frac{p_{i}-1}{p_{i}}} \max \left\{\left(\frac{1}{\left\|a_{i}\right\|_{1}}\right)^{\frac{1}{p_{i}}} ; \frac{\operatorname{diam}(\Omega)}{N^{\frac{1}{p_{i}}}}\left(\frac{p_{i}-1}{p_{i}-N} m(\Omega)\right)^{\frac{p_{i}-1}{p_{i}}} \frac{\left\|a_{i}\right\|_{\infty}}{\left\|a_{i}\right\|_{1}}\right\}
\end{gathered}
$$

for $1 \leq i \leq n$, where $m(\Omega)$ is the Lebesgue measure of the set $\Omega$, and equality occurs when $\Omega$ is a ball. 


\section{Main results}

We state our main result:

Theorem 2. Let $F: \Omega \times \mathbb{R}^{n} \rightarrow \mathbb{R}$ be a function such that $F\left(\cdot, t_{1}, \ldots, t_{n}\right)$ is continuous in $\Omega$ for all $\left(t_{1}, \ldots, t_{n}\right) \in \mathbb{R}^{n}$ and $F(x, \cdot, \ldots, \cdot)$ is $C^{1}$ in $\mathbb{R}^{n}$ for almost every $x \in \Omega$. Assume that there exist a positive constant $r$ and $a$ function $u^{*}=\left(u_{1}^{*}, \ldots, u_{n}^{*}\right) \in X$ such that

$\left(\mathrm{j}_{1}\right) \sum_{i=1}^{n} \frac{\left\|u_{i}^{*}\right\|^{p_{i}}}{p_{i}}>r$

$\left(\mathrm{j}_{2}\right)$ there is a positive constant $\eta$ with

$$
c m(\Omega) \limsup _{\left(\left|t_{1}\right|, \ldots,\left|t_{n}\right|\right) \rightarrow(+\infty, \ldots,+\infty)} \frac{F\left(x, t_{1}, \ldots, t_{n}\right)}{\frac{1}{p_{1}}\left|t_{1}\right|^{p_{1}}+\cdots+\frac{1}{p_{n}}\left|t_{n}\right|^{p_{n}}}<\frac{1}{r \eta}
$$

uniformly with respect to $x \in \Omega$ and satisfying

$$
r \frac{\int_{\Omega} F\left(x, u_{1}^{*}(x), \ldots, u_{n}^{*}(x)\right) d x}{\sum_{i=1}^{n} \frac{\left\|u_{i}^{*}\right\|^{p_{i}}}{p_{i}}}-\int_{\Omega} \max _{\left(t_{1}, \ldots, t_{n}\right) \in K_{1}} F\left(x, t_{1}, \ldots, t_{n}\right) d x>\frac{1}{\eta} .
$$

where $K_{1}=\left\{\left(t_{1}, \ldots, t_{n}\right) \mid \sum_{i=1}^{n} \frac{\left|t_{i}\right|^{p_{i}}}{p_{i}} \leq \mathrm{cr}\right\}$,

$\left(\mathrm{j}_{3}\right) F(x, 0, \ldots, 0)=0$ for almost every $x \in \Omega$.

Then, there exist a non-empty open interval $A \subseteq] 0, r \eta]$ and a positive real number $q$ with the following property: for every $\lambda \in \Lambda$ and an arbitrary function $G: \Omega \times \mathbb{R}^{n} \rightarrow \mathbb{R}$ measurable in $\Omega$ for all $\left(t_{1}, \ldots, t_{n}\right) \in \mathbb{R}^{n}$ and $C^{1}$ in $\mathbb{R}^{n}$ for almost every $x \in \Omega$, there is $\delta>0$ such that, for each $\mu \in[0, \delta]$ problem (1) admits at least three weak solutions in $X$ whose norms are less than $q$.

Let us first give a consequence of Theorem 2 for a fixed test function $u^{*}$.

Corollary 1. Let $F: \Omega \times \mathbb{R}^{n} \rightarrow \mathbb{R}$ be a function such that $F\left(\cdot, t_{1}, \ldots, t_{n}\right)$ is continuous in $\Omega$ for all $\left(t_{1}, \ldots, t_{n}\right) \in \mathbb{R}^{n}$ and $F(x, \cdot, \ldots, \cdot)$ is $C^{1}$ in $\mathbb{R}^{n}$ for almost every $x \in \Omega$ such that Assumption $\left(\mathrm{j}_{3}\right)$ in Theorem 2 holds. Assume that there exist $n+2$ positive constants $\theta_{i}, \tau$ and $\eta$ with $\theta_{i}<\tau$ for $1 \leq i \leq n$ such that

$\left(\mathrm{j}_{4}\right)$

$$
c m(\Omega) \limsup _{\left(\left|t_{1}\right|, \ldots,\left|t_{n}\right|\right) \rightarrow(+\infty, \ldots,+\infty)} \frac{F\left(x, t_{1}, \ldots, t_{n}\right)}{\frac{1}{p_{1}}\left|t_{1}\right|^{p_{1}}+\cdots+\frac{1}{p_{n}}\left|t_{n}\right|^{p_{n}}}<\frac{c}{\eta \sum_{i=1}^{n} \frac{\theta_{i}^{p_{i}}}{p_{i}}}
$$

uniformly with respect to $x \in \Omega$ and

$$
\begin{aligned}
& \frac{1}{c} \frac{\sum_{i=1}^{n} \prod_{j=1, j \neq i}^{n} p_{j} \theta_{i}^{p_{i}}}{\sum_{i=1}^{n} \prod_{j=1, j \neq i}^{n} p_{j} \tau^{p_{i}}\left\|a_{i}\right\|_{1}} \int_{\Omega} F(x, \tau, \ldots, \tau) d x \\
& -\int_{\Omega} \max _{\left(t_{1}, \ldots, t_{n}\right) \in K_{2}} F\left(x, t_{1}, \ldots, t_{n}\right) d x>\frac{1}{\eta}
\end{aligned}
$$

where $K_{2}=\left\{\left(t_{1}, \ldots, t_{n}\right) \mid \sum_{i=1}^{n} \frac{\left|t_{i}\right|^{p_{i}}}{p_{i}} \leq \sum_{i=1}^{n} \frac{\theta_{i}^{p_{i}}}{p_{i}}\right\}$.

Then, there exist a non-empty open interval $\left.A \subseteq] 0, \frac{\eta}{c} \sum_{i=1}^{n} \frac{\theta_{i}^{p_{i}}}{p_{i}}\right]$ and a positive 
real number $q$ with the following property: for every $\lambda \in \Lambda$ and an arbitrary function $G: \Omega \times \mathbb{R}^{n} \rightarrow \mathbb{R}$ measurable in $\Omega$ for all $\left(t_{1}, \ldots, t_{n}\right) \in \mathbb{R}^{n}$ and $C^{1}$ in $\mathbb{R}^{n}$ for almost every $x \in \Omega$, there is $\delta>0$ such that, for each $\mu \in[0, \delta]$ problem (1) admits at least three weak solutions in $X$ whose norms are less than $q$.

Proof. Choose $u^{*}(x)=\left(u_{1}^{*}(x), u_{2}^{*}(x), \ldots, u_{n}^{*}(x)\right)=(\tau, \tau, \ldots, \tau)$ for every $x \in$ $\Omega$. Then we have $\sum_{i=1}^{n} \frac{\left\|u_{i}^{*}\right\|^{p_{i}}}{p_{i}}=\sum_{i=1}^{n} \frac{\tau^{p_{i}}}{p_{i}}\left\|a_{i}\right\|_{1}$. Put $r=\frac{1}{c} \sum_{i=1}^{n} \frac{\theta_{i}^{p_{i}}}{p_{i}}$. Now since $\theta_{i}<\tau$, bearing in mind that $\frac{1}{\left\|a_{i}\right\|_{1}} \leq c$ for $1 \leq i \leq n$, one has $\sum_{i=1}^{n} \frac{\left\|u_{i}^{*}\right\|^{p_{i}}}{p_{i}}>r$ which is $\left(\mathrm{j}_{1}\right)$. Also, since

$$
\begin{aligned}
& r \frac{\int_{\Omega} F\left(x, u_{1}^{*}(x), \ldots, u_{n}^{*}(x)\right) d x}{\sum_{i=1}^{n} \frac{\left\|u_{i}^{*}\right\|^{p_{i}}}{p_{i}}} \\
= & \frac{\frac{1}{c} \sum_{i=1}^{n} \frac{\theta_{i}^{p_{i}}}{p_{i}}}{\sum_{i=1}^{n} \frac{\tau^{p_{i}}}{p_{i}}\left\|a_{i}\right\|_{1}} \int_{\Omega} F\left(x, u_{1}^{*}(x), \ldots, u_{n}^{*}(x)\right) d x \\
= & \frac{\frac{1}{c}\left(\prod_{j=1}^{n} p_{j}\right) \sum_{i=1}^{n} \frac{\theta_{i}^{p_{i}}}{p_{i}}}{\left(\prod_{j=1}^{n} p_{j}\right) \sum_{i=1}^{n} \frac{\tau^{p_{i}}}{p_{i}}\left\|a_{i}\right\|_{1}} \int_{\Omega} F\left(x, u_{1}^{*}(x), \ldots, u_{n}^{*}(x)\right) d x \\
= & \frac{1}{c} \frac{\sum_{i=1}^{n} \prod_{j=1, j_{j \neq}}^{n} p_{j} \theta_{i}^{p_{i}}}{\sum_{i=1}^{n} \prod_{j=1,,_{j \neq i}}^{n} p_{j} \tau^{p_{i}}\left\|a_{i}\right\|_{1}} \int_{\Omega} F(x, \tau, \ldots, \tau) d x,
\end{aligned}
$$

$\left(\mathrm{j}_{4}\right)$ guarantees $\left(\mathrm{j}_{2}\right)$.

Example 1. Consider the problem

$$
\left\{\begin{aligned}
-\Delta_{3} u_{1}+\frac{2\left(x^{2}+y^{2}\right)}{\pi}\left|u_{1}\right| u_{1}= & \lambda\left(x^{2}+y^{2}\right) e^{-u_{1}} u_{1}^{14}\left(15-u_{1}\right) \\
& +\mu G_{u_{1}}\left(x, y, u_{1}, u_{2}, u_{3}\right) \quad \text { in } \Omega \\
-\Delta_{3} u_{2}+\frac{2\left(x^{2}+y^{2}\right)}{\pi}\left|u_{2}\right| u_{2}= & \lambda\left(x^{2}+y^{2}\right) e^{-u_{2}} u_{2}^{14}\left(15-u_{2}\right) \\
& +\mu G_{u_{2}}\left(x, y, u_{1}, u_{2}, u_{3}\right) \quad \text { in } \Omega \\
-\Delta_{3} u_{3}+\frac{2\left(x^{2}+y^{2}\right)}{\pi}\left|u_{3}\right| u_{3}= & \lambda\left(x^{2}+y^{2}\right) e^{-u_{3}} u_{3}^{14}\left(15-u_{3}\right) \\
& +\mu G_{u_{3}}\left(x, y, u_{1}, u_{2}, u_{3}\right) \quad \text { in } \Omega \\
\frac{\partial u_{i}}{\partial v}= & 0 \quad \text { for } 1 \leq i \leq 3
\end{aligned}\right.
$$

where $\Omega=\left\{(x, y) \in \mathbb{R}^{2} ; x^{2}+y^{2}<9\right\}$ and $G: \Omega \times \mathbb{R}^{3} \rightarrow \mathbb{R}$ is measurable in $\Omega$ for all $\left(t_{1}, t_{2}, t_{3}\right) \in \mathbb{R}^{3}$ and is $C^{1}$ in $\mathbb{R}^{3}$ for almost every $x \in \Omega$. Note that $c=\frac{1536}{\pi}$ and we choose $\theta_{i}=1, \tau=10, a_{i}(x, y)=\frac{2\left(x^{2}+y^{2}\right)}{\pi}$ for $1 \leq i \leq 3$ and

$$
F\left(x, y, u_{1}, u_{2}, u_{3}\right)=\left(x^{2}+y^{2}\right) \sum_{i=1}^{3} e^{-u_{i}} u_{i}^{15}
$$


for each $(x, y) \in \Omega$ and $\left(u_{1}, u_{2}, u_{3}\right) \in \mathbb{R}^{3}$. We see that

$$
\max _{u_{1}^{3}+u_{2}^{3}+u_{3}^{3} \leq 3} \sum_{i=1}^{3} e^{-u_{i}} u_{i}^{15} \leq 3 \max _{u_{i}^{3} \leq 3}\left(e^{-u_{i}} u_{i}^{15}\right) \quad \text { for } 1 \leq i \leq 3
$$

and so

$$
\begin{aligned}
& \frac{1}{c} \frac{\sum_{i=1}^{n} \prod_{j=1, j \neq i}^{n} p_{j} \theta_{i}^{p_{i}}}{\sum_{i=1}^{n} \prod_{j=1, j_{j \neq i}}^{n} p_{j} \tau^{p_{i}}\left\|a_{i}\right\|_{1}} \int_{\Omega} F(x, \tau, \tau, \tau) d x \\
& -\int_{\Omega} \max _{\left(t_{1}, t_{2}, t_{3}\right) \in K_{2}} F\left(x, y, t_{1}, t_{2}, t_{3}\right) d x \\
= & \frac{\pi^{2}}{1024} 10^{12} e^{-10}-\frac{81 \pi}{2} \max _{u_{1}^{3}+u_{2}^{3}+u_{3}^{3} \leq 3} \sum_{i=1}^{3} e^{-u_{i}} u_{i}^{15} \\
\geq & \frac{\pi^{2}}{1024} 10^{12} e^{-10}-\frac{243 \pi}{2} \max _{u_{i}^{3} \leq 3}\left(e^{-u_{i}} u_{i}^{15}\right) \quad \text { for } 1 \leq i \leq 3 \\
= & \frac{\pi^{2}}{1024} 10^{12} e^{-10}-\frac{243 \pi}{2} e^{-\sqrt[3]{3}} 3^{5}>0,
\end{aligned}
$$

and

$$
\limsup _{\left(\left|t_{1}\right|,\left|t_{2}\right|,\left|t_{2}\right|\right) \rightarrow(+\infty,+\infty,+\infty)} \frac{F\left(x, y, t_{1}, t_{2}, t_{3}\right)}{\frac{1}{p_{1}}\left|t_{1}\right|^{p_{1}}+\frac{1}{p_{2}}\left|t_{2}\right|^{p_{2}}+\frac{1}{p_{3}}\left|t_{3}\right|^{p_{3}}}=0
$$

since $p_{i}=3$ for $1 \leq i \leq 3$. We can now apply Corollary 1 to problem (6) for every

$$
\eta>\frac{1}{\frac{\pi^{2}}{1024} 10^{12} e^{-10}-\frac{81 \pi}{2} \max _{u_{1}^{3}+u_{2}^{3}+u_{3}^{3} \leq 3} \sum_{i=1}^{3} e^{-u_{i}} u_{i}^{15}} .
$$

We now point out a special situation of Corollary 1 , in which the function $F$ has separated variables.

Corollary 2. Let $f$ be a continuous function in $\Omega$ and $\tilde{f}_{i}$ for $1 \leq i \leq n$ be a function in $C^{1}$ in $\mathbb{R}^{n}$ for almost every $x \in \Omega$. Assume that there exist $n+2$ positive constants $\theta_{i}, \tau$ and $\eta$ for $1 \leq i \leq n$ with $\theta_{i}<\tau$ for $1 \leq i \leq n$ such that

$\left(\mathrm{j}_{5}\right)$

$$
c m(\Omega) f(x) \limsup _{\left(\left|t_{1}\right|, \ldots,\left|t_{n}\right|\right) \rightarrow(+\infty, \ldots,+\infty)} \frac{\prod_{i=1}^{n} \tilde{f}_{i}\left(t_{i}\right)}{\frac{1}{p_{1}}\left|t_{1}\right|^{p_{1}}+\cdots+\frac{1}{p_{n}}\left|t_{n}\right|^{p_{n}}}<\frac{c}{\eta \sum_{i=1}^{n} \frac{\theta_{i}^{p_{i}}}{p_{i}}}
$$

uniformly with respect to $x \in \Omega$ and

$$
\int_{\Omega} f(x) d x\left[\frac{1}{c} \frac{\sum_{i=1}^{n} \prod_{j=1, j_{j \neq i}}^{n} p_{j} \theta_{i}^{p_{i}}}{\sum_{i=1}^{n} \prod_{j=1, j_{j \neq i}}^{n} p_{j} \tau^{p_{i}}\left\|a_{i}\right\|_{1}} \prod_{i=1}^{n} \tilde{f}_{i}(\tau)-\max _{\left(t_{1}, \ldots, t_{n}\right) \in K_{2}} \prod_{i=1}^{n} \tilde{f}_{i}\left(t_{i}\right)\right]>\frac{1}{\eta},
$$

where $K_{2}=\left\{\left(t_{1}, \ldots, t_{n}\right) \mid \sum_{i=1}^{n} \frac{\left|t_{i}\right|^{p_{i}}}{p_{i}} \leq \sum_{i=1}^{n} \frac{\theta_{i}^{p_{i}}}{p_{i}}\right\}$,

( $\left.\mathrm{j}_{6}\right) \tilde{f}_{i}(0)=0$ for $1 \leq i \leq n$.

Then, there exist a non-empty open interval $\left.\Lambda \subseteq] 0, \frac{\eta}{c} \sum_{i=1}^{n} \frac{\theta_{i}^{p_{i}}}{p_{i}}\right]$ and a positive 
real number $q$ with the following property: for every $\lambda \in \Lambda$ and an arbitrary function $G: \Omega \times \mathbb{R}^{n} \rightarrow \mathbb{R}$ measurable in $\Omega$ for all $\left(t_{1}, \ldots, t_{n}\right) \in \mathbb{R}^{n}$ and $C^{1}$ in $\mathbb{R}^{n}$ for almost every $x \in \Omega$, there is $\delta>0$ such that, for each $\mu \in[0, \delta]$ problem

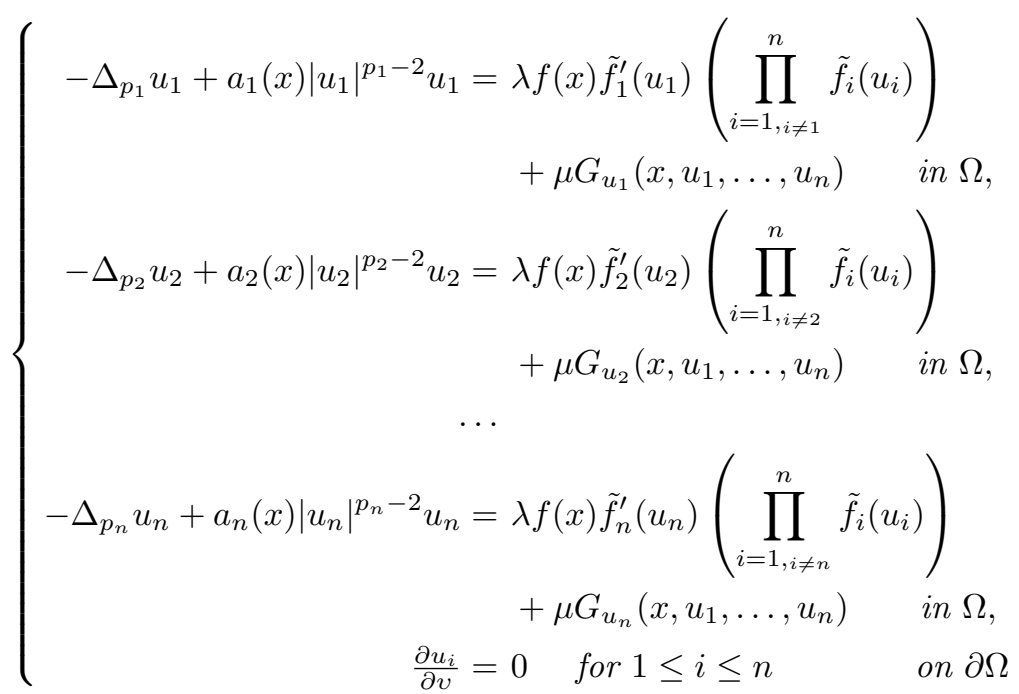

admits at least three weak solutions in $X$ whose norms are less than $q$.

Proof. Set

$$
F\left(x, u_{1}, \ldots, u_{n}\right)=f(x)\left(\prod_{i=1}^{n} \tilde{f}_{i}\left(u_{i}\right)\right)
$$

for each $\left(x, u_{1}, \ldots, u_{n}\right) \in \Omega \times \mathbb{R}^{n}$, and note that

$$
\int_{\Omega} \max _{\left(t_{1}, \ldots, t_{n}\right) \in K_{2}} F\left(x, t_{1}, \ldots, t_{n}\right) d x=\max _{\left(t_{1}, \ldots, t_{n}\right) \in K_{2}} \prod_{i=1}^{n} \tilde{f}_{i}\left(t_{i}\right) \int_{\Omega} f(x) d x
$$

and

$$
\int_{\Omega} F(x, \tau, \ldots, \tau) d x=\prod_{i=1}^{n} \tilde{f}_{i}(\tau) \int_{\Omega} f(x) d x,
$$

and from $\left(\mathrm{j}_{5}\right)$ and $\left(\mathrm{j}_{6}\right)$, it is easy to verify that all the assumptions of Corollary 1 are satisfied. Hence, the proof is complete.

Corollary 3. Let $F: \mathbb{R}^{n} \rightarrow \mathbb{R}$ be a $C^{1}$ function and assume that there exist $n+2$ positive constants $\theta_{i}, \tau$ and $\eta$ for $1 \leq i \leq n$ with $\theta_{i}<\tau$ for $1 \leq i \leq n$ such that

(j7)

$$
c m(\Omega) \limsup _{\left(\left|t_{1}\right|, \ldots,\left|t_{n}\right|\right) \rightarrow(+\infty, \ldots,+\infty)} \frac{F\left(t_{1}, \ldots, t_{n}\right)}{\frac{1}{p_{1}}\left|t_{1}\right|^{p_{1}}+\cdots+\frac{1}{p_{n}}\left|t_{n}\right|^{p_{n}}}<\frac{c}{\eta \sum_{i=1}^{n} \frac{\theta_{i}^{p_{i}}}{p_{i}}}
$$


and

$$
m(\Omega)\left(\frac{1}{c} \frac{\sum_{i=1}^{n} \prod_{j=1, j \neq i}^{n} p_{j} \theta_{i}^{p_{i}}}{\sum_{i=1}^{n} \prod_{j=1, j \neq i}^{n} p_{j} \tau^{p_{i}}\left\|a_{i}\right\|_{1}} F(\tau, \ldots, \tau)-\max _{\left(t_{1}, \ldots, t_{n}\right) \in K_{2}} F\left(t_{1}, \ldots, t_{n}\right)\right)>\frac{1}{\eta},
$$

where $K_{2}=\left\{\left(t_{1}, \ldots, t_{n}\right) \mid \sum_{i=1}^{n} \frac{\left|t_{i}\right|^{p_{i}}}{p_{i}} \leq \sum_{i=1}^{n} \frac{\theta_{i}^{p_{i}}}{p_{i}}\right\}$,

$\left(\mathrm{j}_{8}\right) F(0, \ldots, 0)=0$.

Then, there exist a non-empty open interval $\left.\Lambda \subseteq] 0, \frac{\eta}{c} \sum_{i=1}^{n} \frac{\theta_{i}^{p_{i}}}{p_{i}}\right]$ and a positive real number $q$ with the following property: for every $\lambda \in \Lambda$ and an arbitrary $C^{1}$ function $G: \mathbb{R}^{n} \rightarrow \mathbb{R}$, there is $\delta>0$ such that, for each $\mu \in[0, \delta]$ problem (8)

$$
\left\{\begin{aligned}
&-\Delta_{p_{1}} u_{1}+a_{1}(x)\left|u_{1}\right|^{p_{1}-2} u_{1}=\lambda F_{u_{1}}\left(u_{1}, \ldots, u_{n}\right)+\mu G_{u_{1}}\left(u_{1}, \ldots, u_{n}\right) \text { in } \Omega, \\
&-\Delta_{p_{2}} u_{2}+a_{2}(x)\left|u_{2}\right|^{p_{2}-2} u_{2}=\lambda F_{u_{2}}\left(u_{1}, \ldots, u_{n}\right)+\mu G_{u_{2}}\left(u_{1}, \ldots, u_{n}\right) \quad \text { in } \Omega, \\
& \ldots \\
&-\Delta_{p_{n}} u_{n}+a_{n}(x)\left|u_{n}\right|^{p_{n}-2} u_{n}=\lambda F_{u_{n}}\left(u_{1}, \ldots, u_{n}\right)+\mu G_{u_{n}}\left(u_{1}, \ldots, u_{n}\right) \text { in } \Omega, \\
& \frac{\partial u_{i}}{\partial v}=0 \quad \text { for } 1 \leq i \leq n \quad \text { on } \partial \Omega
\end{aligned}\right.
$$

admits at least three solutions in $X$ whose norms are less than $q$.

We now look at a consequence of Corollary 3 in the ordinary case $p_{i}=$ 2 for $1 \leq i \leq n$. For simplicity, we fix $\Omega=(0,1)$. Note in this situation we have $c=2 \max \left\{\left\|a_{i}\right\|_{1}^{-1},\left\|a_{i}\right\|_{\infty}^{2}\left\|a_{i}\right\|_{1}^{-2} ; \quad 1 \leq i \leq n\right\}$.

Corollary 4. Let $F: \mathbb{R}^{n} \rightarrow \mathbb{R}$ be a $C^{1}$ function and assume that there exist $n+2$ positive constants $\theta_{i}, \tau$ and $\eta$ for $1 \leq i \leq n$ with $\theta_{i}<\tau$ for $1 \leq i \leq n$ such that

(j9)

$$
c m(\Omega) \limsup _{\left(\left|t_{1}\right|, \ldots,\left|t_{n}\right|\right) \rightarrow(+\infty, \ldots,+\infty)} \frac{F\left(t_{1}, \ldots, t_{n}\right)}{\frac{1}{2}\left|t_{1}\right|^{2}+\cdots+\frac{1}{2}\left|t_{n}\right|^{2}}<\frac{4 \tilde{c}}{\eta \sum_{i=1}^{n} \theta_{i}^{2}},
$$

where $\tilde{c}=\max \left\{\left\|a_{i}\right\|_{1}^{-1},\left\|a_{i}\right\|_{\infty}^{2}\left\|a_{i}\right\|_{1}^{-2} ; \quad 1 \leq i \leq n\right\}$ and

$$
\frac{\left(\sum_{i=1}^{n} \theta_{i}^{2}\right) F(\tau, \ldots, \tau)}{2 \tilde{c}\left(\tau^{2} \sum_{i=1}^{n}\left\|a_{i}\right\|_{1}\right)}-\max _{\left(t_{1}, \ldots, t_{n}\right) \in K_{3}} F\left(t_{1}, \ldots, t_{n}\right)>\frac{1}{\eta}
$$

where $K_{3}=\left\{\left(t_{1}, \ldots, t_{n}\right) \mid \sum_{i=1}^{n} t_{i}^{2} \leq \sum_{i=1}^{n} \theta_{i}^{2}\right\}$,

$\left(\mathrm{j}_{10}\right) F(0, \ldots, 0)=0$.

Then, there exist a non-empty open interval $\left.\Lambda \subseteq] 0, \frac{\eta}{4 \tilde{c}} \sum_{i=1}^{n} \theta_{i}^{2}\right]$ and a positive real number $q$ with the following property: for every $\lambda \in \Lambda$ and an arbitrary $C^{1}$ function $G: \mathbb{R}^{n} \rightarrow \mathbb{R}$, there is $\delta>0$ such that, for each $\mu \in[0, \delta]$ such that, 
problem

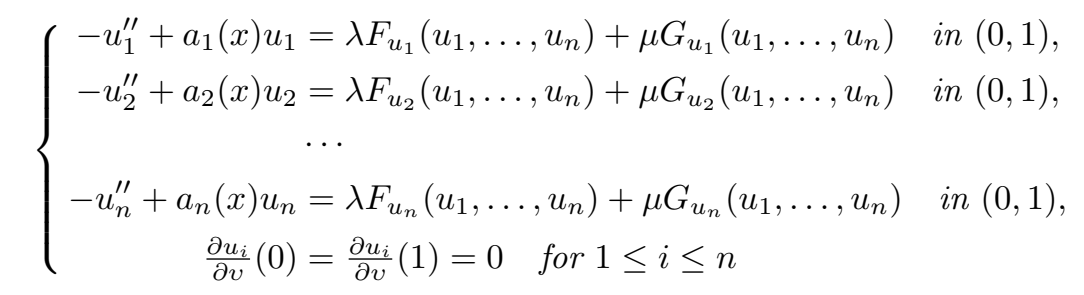

admits at least three weak solutions in $X$ whose norms are less than $q$.

\section{Existence results in the case $n=1$}

Consider the following Neumann elliptic problem

$$
\left\{\begin{aligned}
-\Delta_{p} u+a(x)|u|^{p-2} u_{1} & =\lambda f(x, u)+\mu g(x, u) \quad \text { in } \Omega, \\
\frac{\partial u}{\partial v} & =0 \quad \text { on } \partial \Omega,
\end{aligned}\right.
$$

where $\Omega \subset \mathbb{R}^{N}(N \geq 1)$ is a nonempty bounded open set with a boundary $\partial \Omega$ of class $C^{1}, a \in L^{\infty}(\Omega)$ with $\operatorname{essinf}_{\Omega} a>0, p>N, \lambda, \mu>0$ and $f, g: \Omega \times \mathbb{R} \rightarrow \mathbb{R}$ are two $L^{1}$-Carathéodory functions. Let $X$ be the Sobolev space $W^{1, p}(\Omega)$ equipped with the norm

$$
\|u\|=\left(\int_{\Omega}\left(|\nabla u(x)|^{p}+a(x)|u(x)|^{p}\right) d x\right)^{1 / p} .
$$

Corresponding to $f$ and $g$ we introduce the functions $F: \Omega \times \mathbb{R} \rightarrow \mathbb{R}$ and $G: \Omega \times \mathbb{R} \rightarrow \mathbb{R}$, respectively as follows

$$
F(x, t)=\int_{0}^{t} f(x, \xi) d \xi
$$

and

$$
G(x, t)=\int_{0}^{t} g(x, \xi) d \xi
$$

for each $(x, t) \in \Omega \times \mathbb{R}$. Put

$$
k=\sup _{u \in W^{1, p}(\Omega) \backslash\{0\}} \frac{\max _{x \in \bar{\Omega}}|u(x)|}{\|u\|} .
$$

Since $p>N$, one has $k<+\infty$. It follows from Proposition 4.1 of [3] that $k \geq \frac{1}{\|a\|_{1}^{\frac{1}{p}}}$, where $\|a\|_{1}=\int_{\Omega}|a(x)| d x$.

Now, we present the following result as an application of Corollary 1 . Note that $F(x, 0)=0$ for all $x \in \Omega$.

Corollary 5. Assume that there exist three positive constants $\theta, \tau$ and $\eta$ with $\theta<\tau$ such that 
$\left(\mathrm{j}_{11}\right) m(\Omega) \lim \sup _{|t| \rightarrow+\infty} \frac{F(x, t)}{|t|^{p}}<\frac{1}{\eta \theta^{p}}$ uniformly with respect to $x \in \Omega$ and

$$
\left(\frac{\theta}{k}\right)^{p} \frac{\int_{\Omega} F(x, \tau) d x}{\tau^{p}\|a\|_{1}}-\int_{\Omega} \max _{t \in[-\theta, \theta]} F(x, t) d x>\frac{1}{\eta} .
$$

Then, there exist a non-empty open interval $\left.A \subseteq] 0, \frac{\eta}{p}\left(\frac{\theta}{k}\right)^{p}\right]$ and a positive real number $q$ with the following property: for every $\lambda \in \Lambda$ and an arbitrary $L^{1}$ Carathéodory function $g: \Omega \times \mathbb{R} \rightarrow \mathbb{R}$, there is $\delta>0$ such that, for each $\mu \in[0, \delta]$ problem (10) admits at least three weak solutions in $X$ whose norms are less than $q$.

We conclude this section by giving a simple consequence of Corollary 5 when $N=1$ and $p=2$. For simplicity, we fix $\Omega=(a, b)$. In this case, we have

$$
k=\sqrt{2} \max \left\{\|a\|_{1}^{-\frac{1}{2}},(a+b)(b-a)^{-\frac{1}{2}}\|a\|_{\infty}\|a\|_{1}^{-1}\right\} .
$$

Corollary 6. Assume that there exist three positive constants $\theta, \tau$ and $\eta$ with $\theta<\tau$ such that and

$\left(\mathrm{j}_{12}\right)(b-a) \lim \sup _{|t| \rightarrow+\infty} \frac{F(x, t)}{|t|^{2}}<\frac{1}{\eta \theta^{2}}$ uniformly with respect to $x \in(a, b)$

$$
\left(\frac{\theta}{k}\right)^{2} \frac{\int_{a}^{b} F(x, \tau) d x}{\tau^{2}\|a\|_{1}}-\int_{a}^{b} \max _{t \in[-\theta, \theta]} F(x, t) d x>\frac{1}{\eta} .
$$

Then, there exist a non-empty open interval $\left.A \subseteq] 0, \frac{\eta}{2}\left(\frac{\theta}{k}\right)^{2}\right]$ and a positive real number $q$ with the following property: for every $\lambda \in \Lambda$ and an arbitrary $L^{1}$ Carathéodory function $g:[a, b] \times \mathbb{R} \rightarrow \mathbb{R}$, there is $\delta>0$ such that, for each $\mu \in[0, \delta]$ problem

$$
\left\{\begin{aligned}
-u^{\prime \prime}+a(x) u & =\lambda f(x, u)+\mu g(x, u), \\
u^{\prime}(a) & =u^{\prime}(b)=0
\end{aligned}\right.
$$

admits at least three weak solutions in $X$ whose norms are less than $q$.

\section{Proof of Theorem 1}

We introduce the functionals $\Phi, J: X \rightarrow \mathbb{R}$ for each $u \in X$, as follows

$$
\Phi(u)=\sum_{i=1}^{n} \frac{\left\|u_{i}\right\|^{p_{i}}}{p_{i}},
$$

and

$$
J(u)=-\int_{\Omega} F\left(x, u_{1}(x), \ldots, u_{n}(x)\right) d x .
$$

Note $\Phi$ is bounded on each bounded subset of $X$, it is continuously differentiable and sequentially weakly lower semicontinuous functional, its differential admits a continuous inverse on $X^{*}$ and since $p_{i}>N$ for $1 \leq i \leq n, J$ is a continuously 
differentiable functional with compact derivative. In particular, for each $u=$ $\left(u_{1}, \ldots, u_{n}\right), v=\left(v_{1}, \ldots, v_{n}\right) \in X$ one has

$\Phi^{\prime}(u)(v)=\int_{\Omega} \sum_{i=1}^{n}\left(\left|\nabla u_{i}(x)\right|^{p_{i}-2} \nabla u_{i}(x) \nabla v_{i}(x)+a_{i}(x)\left|u_{i}(x)\right|^{p_{i}-2} u_{i}(x) v_{i}(x)\right) d x$,

and

$$
J^{\prime}(u)(v)=-\int_{\Omega} \sum_{i=1}^{n} F_{u_{i}}\left(x, u_{1}(x), \ldots, u_{n}(x)\right) v_{i}(x) d x .
$$

Furthermore from $\left(\mathrm{j}_{2}\right)$ there exist two constants $\alpha, \beta \in R$ with $0<\alpha<\frac{1}{r \eta}$ such that

$$
c m(\Omega) F\left(x, t_{1}, \ldots, t_{n}\right) \leq \alpha\left(\frac{1}{p_{1}}\left|t_{1}\right|^{p_{1}}+\cdots+\frac{1}{p_{n}}\left|t_{n}\right|^{p_{n}}\right)+\beta
$$

for a.e. $x \in \Omega$ and all $t \in \mathbb{R}$. Fix $u=\left(u_{1}, \ldots, u_{n}\right) \in X$. Then

$$
\begin{aligned}
F\left(x, u_{1}(x), \ldots, u_{n}(x)\right) \leq & \frac{\alpha}{c m(\Omega)}\left(\frac{1}{p_{1}}\left|u_{1}(x)\right|^{p_{1}}+\cdots+\frac{1}{p_{n}}\left|u_{n}(x)\right|^{p_{n}}\right) \\
& +\frac{\beta}{c m(\Omega)} \text { for a.e. } x \in \Omega .
\end{aligned}
$$

Then, for any fixed $\lambda \in] 0, r \eta]$, since

$$
\sup _{x \in \Omega}\left|u_{i}(x)\right|^{p_{i}} \leq c|| u_{i} \|^{p_{i}}
$$

for $1 \leq i \leq n$, from (11), (12) and (13), we have

$$
\begin{aligned}
\Phi(u)+\lambda J(u)= & \sum_{i=1}^{n} \frac{\left\|u_{i}\right\|^{p_{i}}}{p_{i}}-\lambda \int_{\Omega} F\left(x, u_{1}(x), \ldots, u_{n}(x)\right) d x \\
\geq & \sum_{i=1}^{n} \frac{\left\|u_{i}\right\|^{p_{i}}}{p_{i}} \\
& -\frac{\alpha \lambda}{c m(\Omega)}\left(\frac{1}{p_{1}} \int_{\Omega}\left|u_{1}(x)\right|^{p_{1}} d x+\cdots+\frac{1}{p_{n}} \int_{\Omega}\left|u_{n}(x)\right|^{p_{n}} d x\right)-\frac{\lambda \beta}{c} \\
\geq & (1-\alpha r \eta) \sum_{i=1}^{n} \frac{\left\|u_{i}\right\|^{p_{i}}}{p_{i}}-\frac{r \eta \beta}{c},
\end{aligned}
$$

and so

$$
\lim _{\|u\| \rightarrow+\infty}(\Phi(u)+\lambda J(u))=+\infty .
$$

To check the other assumption in Theorem 1 we use Proposition 1 . From $\left(\mathrm{j}_{1}\right)$ and (11) we obtain $\left(\kappa_{1}\right)$. Moreover, from (14) we have

$$
\sup _{x \in \Omega} \sum_{i=1}^{n} \frac{\left|u_{i}(x)\right|^{p_{i}}}{p_{i}} \leq c \sum_{i=1}^{n} \frac{\left\|u_{i}\right\|^{p_{i}}}{p_{i}}
$$


for each $u=\left(u_{1}, \ldots, u_{n}\right) \in X$, and so for each $r>0$

$\Phi^{-1}(]-\infty, r[)=\left\{u=\left(u_{1}, u_{2}, \ldots, u_{n}\right) \in X ; \Phi(u)<r\right\}$

$$
\begin{aligned}
& =\left\{u=\left(u_{1}, u_{2}, \ldots, u_{n}\right) \in X ; \sum_{i=1}^{n} \frac{\|\left. u_{i}\right|^{p_{i}}}{p_{i}}<r\right\} \\
& \subseteq\left\{u=\left(u_{1}, u_{2}, \ldots, u_{n}\right) \in X ; \sum_{i=1}^{n} \frac{\left|u_{i}(x)\right|^{p_{i}}}{p_{i}} \leq c r \text { for each } x \in \Omega\right\} .
\end{aligned}
$$

Now since

$$
r \frac{\int_{\Omega} F\left(x, u_{1}^{*}(x), \ldots, u_{n}^{*}(x)\right) d x}{\sum_{i=1}^{n} \frac{\left\|u_{i}^{*}\right\|^{p_{i}}}{p_{i}}}-\int_{\Omega} \max _{\left(t_{1}, \ldots, t_{n}\right) \in K_{1}} F\left(x, t_{1}, \ldots, t_{n}\right) d x>\frac{1}{\eta}>0
$$

we have

$$
\int_{\Omega} \max _{\left(t_{1}, \ldots, t_{n}\right) \in K_{1}} F\left(x, t_{1}, \ldots, t_{n}\right) d x<r \frac{\int_{\Omega} F\left(x, u_{1}^{*}(x), \ldots, u_{n}^{*}(x)\right) d x}{\sum_{i=1}^{n} \frac{\left\|u_{i}^{*}\right\|^{p_{i}}}{p_{i}}},
$$

and we obtain (see (14))

$$
\begin{aligned}
\sup _{u \in \Phi^{-1}(]-\infty, r[)}(-J(u)) & =\sup _{\sum_{i=1}^{n} \frac{\left\|u_{i}\right\|^{p_{i}}}{p_{i}}<r} \int_{\Omega} F\left(x, u_{1}(x), \ldots, u_{n}(x)\right) d x \\
& \leq \int_{\Omega} \max _{\Omega}\left(t_{1}, \ldots, t_{n}\right) \in K_{1} \\
& <r \frac{\int_{\Omega} F\left(x, u_{1}^{*}(x), \ldots, t_{1}, \ldots, t_{n}\right) d x}{\sum_{i=1}^{n} \frac{\left\|u_{i}^{*}\right\|^{p_{i}}}{p_{i}}} \\
& =r \frac{-J\left(u^{*}(x)\right)}{\Phi\left(u^{*}(x)\right)}
\end{aligned}
$$

where $K_{1}=\left\{\left(t_{1}, \ldots, t_{n}\right) \mid \sum_{i=1}^{n} \frac{\left|t_{i}\right|^{p_{i}}}{p_{i}} \leq c r\right\}$, so

$$
\sup _{u \in \Phi^{-1}(]-\infty, r[)}(-J(u))<r \frac{-J\left(u^{*}\right)}{\Phi\left(u^{*}\right)},
$$

which means that $\left(\kappa_{2}\right)$ is fulfilled. Next recall from $\left(\mathrm{j}_{2}\right)$ that

$$
\eta>\frac{1}{r \frac{-J\left(u^{*}\right)}{\Phi\left(u^{*}\right)}-\sup _{u \in \Phi^{-1}(]-\infty, r[)}(-J(u))} \text {. }
$$

Choose

and note $\nu>1$. Also, since

$$
\nu=\eta\left(r \frac{-J\left(u^{*}\right)}{\Phi\left(u^{*}\right)}-\sup _{u \in \Phi^{-1}(]-\infty, r[)}(-J(u))\right),
$$

$$
\eta>\frac{1}{r \frac{-J\left(u^{*}\right)}{\Phi\left(u^{*}\right)}-\sup _{u \in \Phi^{-1}(]-\infty, r[)}(-J(u))},
$$


we have

$$
\sup _{u \in \Phi^{-1}(]-\infty, r[)}(-J(u))+\frac{1}{\eta}<r \frac{-J\left(u^{*}\right)}{\Phi\left(u^{*}\right)},
$$

and so with our choice of $\nu$ we have

$$
\sup _{u \in \Phi^{-1}(]-\infty, r[)}(-J(u))+\frac{r \frac{-J\left(u^{*}\right)}{\Phi\left(u^{*}\right)}-\sup _{u \in \Phi^{-1}(]-\infty, r[)}(-J(u))}{\nu}<r \frac{-J\left(u^{*}\right)}{\Phi\left(u^{*}\right)} .
$$

Now from Proposition 1 (with $u_{0}=0$ and $u_{1}=u^{*}$ ) for every $\rho \in R$ satisfying

$$
\sup _{\left.\left.u \in \Phi^{-1}(]-\infty, r\right]\right)}(-J(u))+\frac{r \frac{-J\left(u^{*}\right)}{\Phi\left(u^{*}\right)}-\sup _{u \in \Phi^{-1}(]-\infty, r[)}(-J(u))}{\nu}<\rho<r \frac{-J\left(u^{*}\right)}{\Phi\left(u^{*}\right)},
$$

we have (note $\sigma=r \eta$ )

$$
\sup _{\lambda \in R} \inf _{u \in X}(\Phi(u)+\lambda J(u)+\rho \lambda)<\inf _{u \in X} \sup _{\lambda \in[0, r \eta]}(\Phi(u)+\lambda J(u)+\rho \lambda) .
$$

For any fixed function $G: \Omega \times \mathbb{R}^{N} \rightarrow \mathbb{R}$ as in the statement of the theorem, set

$$
\Psi(u)=-\int_{\Omega} G\left(x, u_{1}(x), \ldots, u_{n}(x)\right) d x .
$$

It is well known that $\Psi$ is a continuously differentiable functional whose differential $\Psi^{\prime}(u) \in X^{*}$, at $u \in X$ is given by

$\Psi^{\prime}(u) v=-\int_{\Omega} \sum_{i=1}^{n} G_{u_{i}}\left(x, u_{1}(x), \ldots, u_{n}(x)\right) v_{i}(x) d x$ for every $\left(v_{1}, \ldots, v_{n}\right) \in X$,

such that $\Psi^{\prime}: X \rightarrow X^{*}$ is a compact operator. Now, all the assumptions of Theorem 1, are satisfied. Hence, applying Theorem 1, and taking into account that the critical points of the functional $\Phi+\lambda J+\mu \Psi$ are exactly the weak solutions of the problem (1), we have the conclusion.

\section{References}

[1] G. A. Afrouzi and S. Heidarkhani, Existence of three solutions for a class of Dirichlet quasilinear elliptic systems involving the $\left(p_{1}, \ldots, p_{n}\right)$-Laplacian, Nonlinear Anal. 70 (2009), no. 1, 135-143.

[2] _ Three solutions for a Dirichlet boundary value problem involving the $p$ Laplacian, Nonlinear Anal. 66 (2007), no. 10, 2281-2288.

[3] G. Anello and G. Cordaro, An existence theorem for the Neumann problem involving the p-Laplacian, J. Convex Anal. 10 (2003), no. 1, 185-198.

[4] __ Infinitely many arbitrarily small positive solutions for the Dirichlet problem involving the p-Laplacian, Proc. Roy. Soc. Edinburgh Sect. A 132 (2002), no. 3, 511519.

[5] L. Boccardo and D. Guedes de Figueiredo, Some remarks on a system of quasilinear elliptic equations, NoDEA Nonlinear Differential Equations Appl. 9 (2002), no. 3, 309323.

[6] G. Bonanno, Existence of three solutions for a two point boundary value problem, Appl. Math. Lett. 13 (2000), no. 5, 53-57.

[7] _ Some remarks on a three critical points theorem, Nonlinear Anal. 54 (2003), no. $4,651-665$. 
[8] G. Bonanno and P. Candito, Three solutions to a Neumann problem for elliptic equations involving the p-Laplacian, Arch. Math. (Basel) 80 (2003), no. 4, 424-429.

[9] G. Bonanno and R. Livrea, Multiplicity theorems for the Dirichlet problem involving the p-Laplacian, Nonlinear Anal. 54 (2003), no. 1, 1-7.

[10] Y. Bozhkova and E. Mitidieri, Existence of multiple solutions for quasilinear systems via fibering method, J. Differential Equations 190 (2003), no. 1, 239-267.

[11] P. Candito, Existence of three solutions for a nonautonomous two point boundary value problem, J. Math. Anal. Appl. 252 (2000), no. 2, 532-537.

[12] A. Djellit and S. Tas, Quasilinear elliptic systems with critical Sobolev exponents in $\mathbb{R}^{N}$, Nonlinear Anal. 66 (2007), no. 7, 1485-1497.

[13] _ On some nonlinear elliptic systems, Nonlinear Anal. 59 (2004), no. 5, 695-706.

[14] P. Drábek, N. M. Stavrakakis, and N. B. Zographopoulos, Multiple nonsemitrivial solutions for quasilinear elliptic systems, Differential Integral Equations 16 (2003), no. 12, 1519-1531.

[15] S. Heidarkhani and D. Motreanu, Multiplicity results for a two-point boundary value problem, preprint.

[16] A. Kristály, Existence of two non-trivial solutions for a class of quasilinear elliptic variational systems on strip-like domains, Proc. Edinb. Math. Soc. (2) 48 (2005), no. $2,465-477$.

[17] C. Li and C.-L. Tang, Three solutions for a class of quasilinear elliptic systems involving the $(p, q)$-Laplacian, Nonlinear Anal. 69 (2008), no. 10, 3322-3329.

[18] S. A. Marano and D. Motreanu, On a three critical points theorem for non-differentiable functions and applications to nonlinear boundary value problems, Nonlinear Anal. 48 (2002), no. 1, Ser. A: Theory Methods, 37-52.

[19] B. Ricceri, Existence of three solutions for a class of elliptic eigenvalue problems, Math. Comput. Modelling 32 (2000), no. 11-13, 1485-1494.

[20] _ On a three critical points theorem, Arch. Math. (Basel) 75 (2000), no. 3, 220226.

[21] _ A three critical points theorem revisited, Nonlinear Anal. 70 (2009), no. 9, 3084-3089.

[22] T. Teramoto, On positive radial entire solutions of second-order quasilinear elliptic systems, J. Math. Anal. Appl. 282 (2003), no. 2, 531-552.

[23] G. Q. Zhang, X. P. Liu, and S. Y. Liu, Remarks on a class of quasilinear elliptic systems involving the $(p, q)$-Laplacian, Electron. J. Differential Equations 2005 (2005), no. 20, $10 \mathrm{pp}$.

Ghasem A. Afrouzi

Department of Mathematics

Faculty of Basic Sciences

UNIVERSITY OF MAZANDARAN

47416-1467 BABOLSAR, IRAN

E-mail address: afrouzi@umz.ac.ir

Shapour Heidarkhani

Department of Mathematics

Faculty of Basic Sciences

RAZI UNIVERSITY

67149 Kermanshah, Iran

E-mail address: sh.heidarkhani@yahoo.com 
1250 GHASEM A. AFROUZI, SHAPOUR HEIDARKHANI, AND DONAL O'REGAN

\author{
Donal O'Regan \\ Department of Mathematics \\ NATional University of IRELAND \\ GalWay, IRELAND \\ E-mail address: donal.oregan@nuigalway.ie
}

